\title{
Tratamiento de infecciones fúngicas sistémicas Primera parte: fluconazol, itraconazol y voriconazol
}

\author{
ALBERTO FICA C.
}

\author{
Treatment of systemic fungal infections. First part: Fluconazole, itraconazole \\ and voriconazole
}

Randomized clinical trials in systemic antifungal therapy have traditionally been focused on patients with systemic candidiasis, cryptococcal meningitis, invasive aspergillosis and febrile neutropenic patients with suspicion of a fungal infection. The latter is a known risk factor for infection with filamentous fungi. Itraconazole and voriconazole are active against molds when compared with fluconazole but they are not active against agents of mucormycosis. Both itraconazole and voriconazole cover clinical isolates of Candida albicans and non C. albicans isolates with natural or acquired resistance to fluconazole. Although every triazole compound can be applied on infection caused by $C$. albicans, only fluconazole has been validated in this setting. Moreover, this compound is the only one approved for the treatment of patients affected by low-risk cryptococcal meningitis. In patients with invasive aspergillosis, only voriconazole has been formally compared and found similar to amphotericin B although open-label studies with intravenous itraconazole also demonstrate its usefulness in this condition. In contrast, in neutropenic febrile patients with suspicion of a fungal infection, intravenous itraconazole but not voriconazole has been shown equivalent to amphotericin B. Triazole compounds differ each other in their dosing schedules, bioavailability, interference of food or changes in gastric $\mathrm{pH}$ in absorption, penetration to the CNS, time to reach plasma steady-state concentrations and acquisition costs. Intravenous presentations of itraconazole and voriconazole should not be administered when moderate or mild renal failure is present $(<30 \mathrm{vs}<50 \mathrm{ml} / \mathrm{min}$ of creatinine clearence, respectively).

Key words: Invasive mycosis, Systemic antifungal agents, Fluconazole, Itraconazole, Voriconazole.

\section{Introducción}

Fluconazol, el compuesto triazólico más conocido, es una alternativa terapéutica habitual en el tratamiento de infecciones fúngicas sistémicas específicas. Recientemente han emergido nuevos triazoles que han ampliado la gama terapéutica disponible. Estos compuestos se caracterizan por un anillo de 5 átomos con 3 de nitrógeno e incluyen además a itraconazol, voriconazol, posaconazol y ravuconazol ${ }^{1}$.

Este artículo ha sido preparado para orientar al lector sobre las ventajas y desventajas asociadas a estos fármacos y sus indicaciones en el tratamiento de las infecciones fúngicas sistémicas (diseminadas o invasoras). Para los propósitos de esta revisión, se entiende por infección fúngica diseminada aquella que ocurre en dos sitios u órganos separados, e infección invasora aquella asociada a un sitio normalmente estéril o a una lesión tisular profunda. No se incluye en esta revisión el uso profiláctico de estos compuestos.

\section{Mecanismo de acción}

Estos compuestos actúan mediante la inhibición de la enzima lanosterol 14- $\alpha$ demetilasa en el complejo citocromo P-450 de los hongos. El resultado es la inhibición de la conversión de lanosterol a ergosterol, con la consecuente depleción de ergosterol, acumulación de precursores y una pérdida de la integridad de la membrana fúngica. ${ }^{1}$ Los imidazoles y en grado variable los triazoles, también interactuan con el complejo P-450 de la especie humana, provocando interferencias metabólicas con ciertas hormonas o interacciones con fármacos metabolizados bajo este sistema.

Sección de Infectología, Departamento de Medicina. Hospital Clínico Universidad de Chile.

Recibido: 29 septiembre 2003

Aceptado: 3 diciembre 2003 


\section{Semejanzas y diferencias}

Los compuestos triazólicos se caracterizan por un mayor espectro de acción que los imidazoles, una adecuada penetración en diferentes fluidos (aunque variable en el SNC), su disponibilidad paralela en formulaciones parenterales $\mathrm{u}$ orales, su seguridad y una menor interacción con el sistema citocromo P-450 humano (Tabla 1). Este último aspecto evita la interferencia sobre la síntesis de cortisol o testosterona pero retiene interacciones importantes con otros fármacos metabolizados por el sistema P-450 hepático o intestinal que limita el uso o contraindica la utilización de otros compuestos debido a las modificaciones que ejercen sobre el nivel plasmático de ellas. ${ }^{2}$

Estos compuestos presentan a su vez diferencias importantes en su biodisponibilidad, espectro sobre hongos filamentosos, penetración al SNC y ajustes de dosis, entre otros aspectos (Tabla 1).

Los preparados triazólicos requieren una dosis de carga para lograr concentraciones plasmáticas estacionarias y terapéuticas; sin embargo, la rapidez con que ello se obtiene varía (Tabla 2). La dosis de carga de fluconazol y voriconazol, equivalente al doble de las dosis de mantención, permite concentraciones estacionarias desde el segundo día. Itraconazol se caracteriza por una lenta obtención de concentraciones estacionarias para su preparado original en cápsulas, que se extiende a 2 semanas. Las nuevas formulaciones como la solución oral (SO) y la presentación parenteral aceleran esta ventana a unos 4 a 8 días y 48 horas, respectivamente.

\section{Interacciones de los compuestos triazólicos con otros fármacos}

Los compuestos imidazólicos y triazólicos, inhiben el metabolismo de otros compuestos debido a su interferencia con diferentes isoenzimas
Tabla 1. Características comunes y diferencias entre triazoles

\section{Aspectos comunes}

Mecanismo de acción

Actividad sobre levaduras prevalentes en infecciones humanas

Efecto fungistático sobre levaduras

Presentaciones orales y endovenosas disponibles

Interacciones con numerosos fármacos

Índice terapéutico elevado

Dosis de carga inicial requerida

\section{Diferencias}

Biodisponibilidad

Grado de interferencia de los alimentos sobre la absorción

\section{Dosificación}

Rapidez en la obtención de concentraciones plasmáticas estacionarias

Penetración al SNC

Ajustes requeridos en presencia de falla renal o hepática

Espectro sobre hongos filamentosos

Costo de adquisición

del complejo citocromo P-450 del ser humano. El resultado neto es un potencial aumento de los niveles plasmáticos de otros fármacos facilitando la aparición de efectos adversos. Sin embargo, la intensidad de este efecto depende del compuesto en particular y de las isoenzimas inhibidas ${ }^{2}$. En un determinado paciente coexisten al menos 3 sistemas citocromales P-450 (CYP1, CYP2 y CYP3) pudiendo tener cada uno de ellos diferentes isoenzimas. Las isoenzimas de mayor importancia en el metabolismo de diferentes fármacos son

Tabla 2. Dosis de carga y de mantención de diferentes preparados triazólicos para infecciones sistémicas

\begin{tabular}{lll}
\hline Compuesto y presentación & \multicolumn{1}{c}{ Dosis de carga y duración } & Dosis de mantención \\
\hline Fluconazol oral & $800 \mathrm{mg} /$ día por un día (en una dosis) & $400 \mathrm{mg} /$ día (una dosis) \\
Fluconazol ev & Idem & Idem \\
Itraconazol cápsulas & $100 \mathrm{a} 200 \mathrm{mg} /$ día por 2 semanas & $100 \mathrm{mg} \mathrm{c} / 12 \mathrm{~h}$ \\
Itraconazol SO* & $400 \mathrm{mg} /$ día por 4 a 8 días & $400 \mathrm{mg} /$ día \\
Itraconazol ev & $200 \mathrm{mg} \mathrm{c} / 12 \mathrm{~h}$ por 48 horas & $200 \mathrm{mg} /$ día (una dosis) \\
Voriconazol oral & $400 \mathrm{mg} \mathrm{c} / 12 \mathrm{~h}$ por 24 horas & $200 \mathrm{mg} \mathrm{c} / 12 \mathrm{~h}$ \\
Voriconazol ev & $6 \mathrm{mg} / \mathrm{kg} \mathrm{c} / 12 \mathrm{~h}$ por 1 día & $4 \mathrm{mg} / \mathrm{kg} \mathrm{c/12} \mathrm{h}$ \\
\hline
\end{tabular}

*SO: solución oral 
las CYP1A2 (15\% de la actividad hepática), CYP2C9, CYP2C19 (que explican el 20\% de la actividad hepática) y CYP3A4, siendo ésta última la isoenzima más abundante y que explica el $30 \%$ de la actividad en el hígado ${ }^{2}$.

Las interacciones de mayor relevancia reportadas para estos compuestos corresponden a la inhibición sobre el metabolismo de benzodiazepinas, ciclosporina, analgésicos opiáceos, fenitoína, digoxina, anticoagulantes orales, ciertos antagonistas del calcio como felodipino, algunos compuestos antiarrítmicos como la quinidina, glibenclamida, antihistamínicos no sedantes (terfenadina, astemizol, loratadina), glucocorticoides y medicamentos antiretrovirales como ritonavir, saquinavir y nevirapina ${ }^{2,3}$ (Tabla 3 ).

Fluconazol inhibe preferentemente a los fármacos metabolizados por las isoenzimas del tipo CYP2C (por ejemplo fenitoína) y, sólo en altas dosis, a los compuestos metabolizados por isoenzima del tipo CYP3 (por ejemplo CYP3A4). Por ello, la interacción de este antifúngico sobre carbamazepina es menos frecuente que sobre fenitoína ya que la carbamazepina se metaboliza preferentemente por la isoenzima CYP3A4, la que es inhibida por fluconazol sólo en altas dosis $\mathrm{s}^{2-5}$.

Los niveles plasmáticos de los compuestos triazólicos pueden disminuir cuando otros compuestos aumentan significativamente la degradación de ellos, arriesgando un fracaso terapéutico.
Ello ocurre con inductores del sistema microsomal hepático tales como rifampicina, fenitoína o carbamazepina (Tabla 3) ${ }^{3,6}$. En el caso de rifampicina, las concentraciones de fluconazol pueden reducirse en forma significativa (> 25\%).

\section{Espectro de acción}

Itraconazol y voriconazol son más activos que fluconazol contra aislados de Candida albicans, Candida no albicans, Cryptococcus neoformans y también son activos contra aislados de $C$. albicans que desarrollan resistencia adquirida a fluconazol o aislados de Candida krusei, caracterizados por una resistencia natural a este compuesto (Tabla 4) ${ }^{7}$.

Itraconazol y voriconazol son más activos que anfotericina B contra Aspergillus fumigatus y A. flavus y equivalentes a anfotericina B contra $A$. niger. Los dos compuestos triazólicos y caspofungina son activos contra $A$. terreus, un microorganismo sobre el cual anfotericina B no demuestra actividad. Sólo voriconazol presenta actividad frente a Scedosporium apiospermum. Tanto voriconazol como anfotericina B poseen actividad moderada contra Fusarium spp (Tabla 5) ${ }^{7-10}$.

A continuación se revisan los compuestos específicos.

\section{Fluconazol}

Este compuesto tiene una alta biodisponibilidad

Tabla 3. Interacciones de importancia clínica con compuestos triazólicos

\begin{tabular}{|c|c|}
\hline Efecto & Compuestos específicos \\
\hline $\begin{array}{l}\text { Fármacos que interfieren en la absorción de } \\
\text { triazoles específicos }\end{array}$ & $\begin{array}{l}\text { - Omeprazol y ranitidina ante itraconazol cápsulas } \\
\text { (incrementan } \mathrm{pH})\end{array}$ \\
\hline $\begin{array}{l}\text { Triazólicos como inhibidores del metabolismo } \\
\text { de otros fármacos }\end{array}$ & $\begin{array}{l}\text { - Benzodiazepinas } \\
\text { - } \text { Aiclosporina } \\
\text { - } \text { Analgésicos opiáceos } \\
\text { - Digoxina } \\
\text { - Anticoagulantes orales } \\
\text { - Quinidina } \\
\text { - Antibenclamida } \\
\text { - Astemizol) } \\
\text { - Glucocorticos no sedantes (loratadina, terfenadina, } \\
\text { - Busulfán } \\
\text { - Buspirona (metina }\end{array}$ \\
\hline Compuestos que aumentan degradación de triazoles & $\begin{array}{l}\text { - Rifampicina } \\
\text { - Fenitoína } \\
\text { - Carbamazepina }\end{array}$ \\
\hline
\end{tabular}


Tabla 4. Actividad comparada de compuestos triazólicos contra diferentes especies de levaduras de importancia clínica $\left(\mathrm{CIM}_{90} \text { en } \mu \mathrm{g} / \mathrm{mL}\right)^{*}$

\begin{tabular}{|c|c|c|c|c|c|}
\hline Compuesto & C. albicans & $\begin{array}{l}\text { C. tropicalis y } \\
\text { C. parapsilosis }\end{array}$ & $\begin{array}{c}\text { C. glabrata, } \\
\text { C. krusei }\end{array}$ & $\begin{array}{l}\text { C. albicans } \\
\text { resistente a } \\
\text { fluconazol }\end{array}$ & C. neoformans \\
\hline Fluconazol & $0,25-8$ & $1-8$ & $8 \quad->128 * *$ & $\geq 128$ & -16 \\
\hline Itraconazol & $0,12-0,25$ & $0,12-0,5$ & $0,25-4$ & 1 & $0,5-1$ \\
\hline Voriconazol & $0,015-0,5$ & $0,03-0,25$ & $0,25-2$ & 1 & $0,12-0,25$ \\
\hline
\end{tabular}

*: Datos tomados de referencia 7; **: C. glabrata 8 a $64 \mu \mathrm{g} / \mathrm{mL}$, C. krusei 64 a $>128 \mu \mathrm{g} / \mathrm{mL}$

Tabla 5. Susceptibilidad in vitro de diferentes especies de hongos filamentosos a compuestos triazólicos, anfotericina $B$ y caspofungina. Valores expresados en $\mathrm{CIM}_{90}$ o media geométrica $(\mu \mathrm{g} / \mathrm{mL})$

\begin{tabular}{|c|c|c|c|c|}
\hline Especie & $\begin{array}{c}\text { Itraconazol } \\
\text { CIM }_{90}\end{array}$ & $\begin{array}{c}\text { Voriconazol } \\
\text { CIM }_{90} \\
\end{array}$ & $\begin{array}{c}\text { Anfotericina B } \\
\text { CIM }_{90}\end{array}$ & $\begin{array}{c}\text { Caspofungina } \\
\text { Media geométrica }\end{array}$ \\
\hline A. fumigatus & $0,5-1$ & $0,25-2$ & -4 & $0,19-2,15$ \\
\hline A. flavus & $0,25-1$ & $0,5-2$ & -8 & $0,5-2,75$ \\
\hline A. niger & $0,5-4$ & $0,5-4$ & $0,125-4$ & $0,04-0,4$ \\
\hline A. terreus & $0,125-0,25$ & 1 & $4 \quad->16$ & $0,2-0,5$ \\
\hline S. apiospermum & $4 \quad->16$ & $0,25-2$ & $->16$ & $4-8 * *$ \\
\hline F. solani & $>16$ & $4->8$ & -4 & $>16 * * *$ \\
\hline
\end{tabular}

Datos tomados de referencias 7 y8, **: datos tomados de referencia 9 y expresados como CIM $* * *$ : datos tomados de referencia 10 y expresados como CIM

luego de su administración oral, alcanzado concentraciones plasmáticas $\geq 80 \%$ de las obtenidas en comparación a una dosis endovenosa. No interfieren en su absorción los inhibidores $\mathrm{H} 2$ o los alimentos en forma significativa y su prolongada vida media permite una dosificación diaria. Este compuesto presenta una farmacocinética lineal, permitiendo mayores concentraciones plasmáticas cuando se incrementa la dosis aplicada. Las concentraciones logradas en el LCR son aproximadamente $70 \%$ de la plasmática. Debido a su alta biodisponibilidad, la mayor parte de los pacientes pueden ser tratados por vía oral con un ahorro importante de recursos. ${ }^{11}$

Las limitaciones más importantes de fluconazol están referidas a su falta de actividad contra hongos filamentosos, a la resistencia natural de algunas levaduras contra este compuesto $(C$. krusei), a la resistencia adquirida en ciertas especies de Candida o $C$. neoformans, a los ajustes de dosis necesarios en pacientes con falla renal y a las interacciones con otros medicamentos (vide supra) (Tabla 6).

La actividad de fluconazol es universal contra aislados de $C$. albicans y $C$. neoformans var. neo-
Tabla 6. Resumen de las principales características de fluconazol

Farmacocinética lineal

Elevada biodisponibilidad

Elevada penetración al SNC (> 70\% en LCR)

Puede ser utilizado en falla renal con ajuste de dosis

Eficacia clínica comprobada en candidemia en ausencia de neutropenia, pacientes con meningitis criptococóccica de bajo riesgo y una variedad de otras infecciones asociadas a levaduras

Bajo costo de adquisición para la presentación oral

formans en nuestro medio ${ }^{12,13}$. Sin embargo, $40 \%$ de los aislados de $C$. glabrata son resistentes a este compuesto al igual que $17 \%$ de C. tropicalis. $^{12}$ La frecuencia de aislados de $C$. albicans o $C$. neoformans con susceptibilidad dosis dependiente (SDD) es aún muy baja en nuestro medio (4 y $10 \%$, respectivamente) ${ }^{12}$ lo que podría generar un fracaso clínico con el uso de las dosis habituales de fluconazol (6 mg/kg/día). Afortunadamente la 
farmacocinética lineal de este antifúngico permite solucionar este fenómeno mediante un aumento de la dosis (12 mg/kg/día).

Debido a que fluconazol es el único preparado triazólico que se excreta mayoritariamente por el riñón, las dosis deben ser ajustadas en pacientes con falla renal. La dosis debe ser reducida a la mitad con cifras de clearance de creatinina $\leq 50$ $\mathrm{ml} / \mathrm{min}$ y a $25 \%$ con cifras $\leq 20 \mathrm{ml} / \mathrm{min}$. Si el paciente está en diálisis, la dosis debe ser administrada después de ella. La remoción de fluconazol es significativamente superior en un proceso de hemodiafiltración en comparación a un proceso de filtración aislada y es recomendable el uso de dosis mayores $(400 \text { a } 800 \mathrm{mg} / \text { día })^{14}$.

La biodisponibilidad de fluconazol no se altera al ser administrado mediante catéteres gástricos o enterales en pacientes con cirugía gastrointestinal $^{15-18}$.

\section{Indicaciones para el uso de fluconazol en infecciones invasoras o diseminadas}

Las indicaciones aprobadas para el uso de fluconazol en infecciones fúngicas sistémicas corresponden al tratamiento de pacientes con candidiasis sistémica sin neutropenia asociada, y al manejo de algunos pacientes afectados por meningitis criptococóccica. ${ }^{19,20}$

Candidemia. La eficacia de fluconazol ha sido demostrada en al menos dos estudios randomizados controlados, comparativos, contra anfotericina $\mathrm{B}$ deoxicolato, antifúngico disponible históricamente para el manejo de las infecciones diseminadas por Candida spp. antes del advenimiento de fluconazol. ${ }^{19,20}$ En el primero de estos ensayos, que excluyó pacientes neutropénicos y en todos los casos se documentó candidemia, se utilizó una dosis de $400 \mathrm{mg} /$ día (inicialmente por vía ev) y durante al menos 14 días, alcanzando éxito terapéutico en $70 \%$, similar a la observada con anfotericina B. La toxicidad global fue menor en el grupo con fluconazol debido a la reducción significativa de los efectos adversos renales o hidroelectrolíticos. El aumento de enzimas hepáticas fue observado con igual frecuencia en ambos grupos $(\sim 10 \%)^{19}$. En este trabajo no se incluyeron pacientes con neoplasias hematológicas y la mayor parte de los casos estuvo asociada a catéteres venosos centrales. Este ensayo permitió establecer como estándar de manejo terapéutico de infecciones diseminadas por Candida, a fluconazol, cuando este tipo de infecciones no está asociada a neutropenia.

El uso de mayores dosis de fluconazol (800 $\mathrm{mg}$ /día) junto a anfotericina $\mathrm{B}$ deoxicolato no ha demostrado un mayor porcentaje de respuestas exitosas o una disminución de la mortalidad en el manejo de pacientes con candidemia no asociada a neutropenia, que las obtenidas en estudios previos con fluconazol a menores dosis $(400 \mathrm{mg}$ diarios $)^{20}$. Sin embargo, la terapia combinada permitió un control más eficaz sobre la candidemia persistente y se observó una tendencia a una respuesta más exitosa en pacientes con un puntaje APACHE II intermedio (10 a 25) pero no en pacientes con puntajes extremos. El tratamiento combinado obligó a reducir las dosis de anfotericina B en $23 \%$ de los casos y las reacciones adversas fueron más frecuentes en el brazo combinado del estudio. Este estudio pone de relieve las dificultades para incrementar la respuesta terapéutica en infecciones asociadas a aislados habitualmente susceptibles a fluconazol, que no requieren estrictamente de un aumento de la dosis y donde la adición de anfotericina B no aporta un beneficio en pacientes con una enfermedad muy leve o muy grave.

Meningitis criptococóccica. La mayor parte de los casos de meningitis criptococóccica se asocia a pacientes con SIDA con una fracción importante que recae luego de la respuesta inicial. El tratamiento inicial comprende una etapa de inducción, que habitualmente dura dos semanas y permite la estabilización o mejoría inicial del paciente, y una etapa de consolidación que dura varias semanas y permite la recuperación progresiva. ${ }^{21}$ Luego de la respuesta inicial los pacientes deben recibir una quimioprofilaxis secundaria en forma crónica. La eficacia de fluconazol en el tratamiento de esta condición debe por lo tanto analizarse en forma separada para las fases de inducción, consolidación y profilaxis secundaria.

En el tratamiento de inducción, fluconazol es igual de eficaz que anfotericina $B$ en pacientes con meningitis criptococóccica sin compromiso de conciencia inicial (bajo riesgo) ${ }^{22}$. En contraste, la tasa de mortalidad precoz es más alta con fluconazol que con anfotericina $B$ en pacientes de alto riesgo (con compromiso de conciencia). (15 versus $8 \%$ ) La respuesta para ambos compuestos es similar en pacientes con altos títulos de antígeno (> 1:1024) de C. neoformans o una baja celularidad en el LCR $\left(<20 \mathrm{cél} / \mathrm{mm}^{3}\right.$, [pobre respuesta inflamatoria]) siempre y cuando no presenten compromiso de conciencia ${ }^{22}$. La aplicación de fluconazol se asocia a una menor frecuencia de reacciones adversas en comparación con anfotericina B y por ello, representa el compuesto de elección en la terapia de inducción en pacientes de bajo riesgo. En el estudio original las dosis de anfotericina $\mathrm{B}$ y de fluconazol fueron 
bajas y ello podría explicar la alta tasa global de fracaso observada (> 50\%). La mayor parte de los pacientes recibió una dosis diaria de $200 \mathrm{mg}$ y sólo un tercio $400 \mathrm{mg}$ diarios. Experiencias posteriores con dosis más altas, aunque no controladas, han permitido establecer que la dosis de inducción con fluconazol debe ser mayor $(\geq 400$ $\mathrm{mg} /$ día ${ }^{23}$. Estas dosis permiten un alta tasa de respuesta (> 60\%) y dosis apropiadas en el LCR, superiores a las CIM reportadas para $C$. neoformans.

Fluconazol es el fármaco de elección en la fase de consolidación cuando los pacientes han respondido favorablemente o se han estabilizado en la fase de inducción ${ }^{21}$. Ello se debe a la posibilidad de su administración oral, a la excelente biodisponibilidad oral, a la obtención de concentraciones apropiadas en el LCR, a su dosificación diaria y a su excelente tolerancia, aspectos que permiten un manejo simplificado en estos pacientes. En estudios comparativos, fluconazol ha resultado superior a itraconazol en la fase de consolidación para lograr una esterilización más rápida del LCR ${ }^{21}$.

Fluconazol también se ha convertido en el medicamento de elección en la quimioprofilaxis secundaria de pacientes recuperados de un evento de meningitis criptococóccica. Una dosis diaria de $200 \mathrm{mg}$ permite el control sobre el riesgo de recaída en más de $90 \%$ de los pacientes, siendo esta cifra superior a la lograda con una dosis semanal de anfotericina B (18\%) y con una menor tasa de efectos adversos ${ }^{24}$.

Otras infecciones. Este compuesto ha sido utilizado en una diversidad de infecciones diseminadas o profundas asociadas a levaduras, como por ejemplo endocarditis de válvula protésica, osteomielitis, infección urinaria, endoftalmitis, infecciones abdominales, etc ${ }^{25,26}$. El conocimiento sobre la eficacia de este compuesto proviene mayoritariamente de los resultados observados en diferentes series de casos.

Este compuesto acelera la esterilización de la orina en pacientes con ITU por Candida sp con o sin sonda Foley pero no previene la recurrencia a corto plazo luego de la interrupción del tratamiento ${ }^{25}$.

Fluconazol ha sido utilizado exitosamente en el manejo de algunos pacientes con endocarditis por Candida asociada a válvula protésica cuando se ha contraindicado la cirugía y el uso de anfotericina B se ha asociado a falla renal ${ }^{26}$.

Otros aspectos. Las dosis habituales utilizadas en infecciones sistémicas varían entre 400 y 800 mg diarios (Tabla 2). Fluconazol debe ser administrado con una dosis de carga equivalente al doble de la terapia a utilizar para asegurar concentraciones estacionarias desde el segundo día. El incremento de la dosis aumenta el riesgo de neurotoxicidad, la que es observada con concentraciones plasmáticas valle $\geq 80 \mu \mathrm{g} / \mathrm{ml}$ y asociado a dosis $\geq 800 \mathrm{mg} /$ día o a dosis menores en presencia de falla renal. ${ }^{27}$

Fluconazol no puede ser utilizado cuando se sospeche o se haya comprobado la existencia de una infección invasora por hongos filamentosos (por ejemplo episodios de neutropenia y fiebre) o levaduras con resistencia natural o adquirida a fluconazol. En casos de infecciones por levaduras con SDD debe evaluarse si es posible utilizar mayores dosis en el paciente. En la Tabla 6 se presenta un resumen de las principales características de fluconazol.

\section{Itraconazol}

Itraconazol es un triazol con espectro sobre diferentes especies de Candida y Aspergillus, disponible en presentaciones orales y endovenosa, es bien tolerado y al parecer induce menos resistencia que fluconazol ${ }^{28}$.

Fue inicialmente introducido en una presentación oral en cápsulas, con baja biodisponibilidad y que sólo permitía lograr concentraciones plasmáticas de equilibrio a los 14 días de administración. Para mejorar su absorción, altamente dependiente de un $\mathrm{pH}$ ácido en el estómago, las cápsulas deben ser ingeridas después de las comidas y en ausencia de antiácidos o inhibidores de la producción de ácido como omeprazol o ranitida ${ }^{6,29-31}$. Su absorción es limitada en pacientes con cirrosis hepática debido a su condición de ayuno, en pacientes neutropénicos por la mala tolerancia digestiva o uso de inhibidores $\mathrm{H} 2$ y en pacientes con SIDA por la hipoclorhidria asocia$\mathrm{da}^{32}$. A pesar de su espectro sobre Aspergillus y su presentación oral que lo convertía en un producto terapéutico atractivo, la formulación disponible no permitía una absorción adecuada, incluso en condiciones óptimas de administración, lo que limitó el uso terapéutico de este compuesto en el manejo de pacientes neutropénicos $u$ otros inmunocomprometidos. Además, muchos de estos pacientes no pueden ingerir adecuadamente los medicamentos orales o presentan intolerancia digestiva hacia ellos. Contribuyó a este segundo plano terapéutico, la imposibilidad de completar un estudio comparativo entre itraconazol y anfotericina B para el manejo de pacientes con aspergilosis invasora y los pobres resultados obtenidos con el uso de este compuesto en estudios abiertos no randomizados para el tratamiento de esta misma condición. ${ }^{33,34}$ 
No obstante estas limitaciones, esta presentación permitía lograr concentraciones terapéuticas apropiadas para infecciones micóticas superficiales en el tracto genital, piel, uñas o esófago y por ello se convirtió en una alternativa para el manejo de vaginitis o esofagitis por Candida y en el manejo de pacientes con tiña u onicomicosis en atención primaria ${ }^{28}$.

Este compuesto ha resurgido por el diseño de formulaciones mejoradas que permiten mejorar la absorción oral o su administración endovenosa $^{28,35}$. Para lograr este objetivo se ha combinado la molécula de itraconazol con un anillo de ciclodextrina, un polímero de glucosa substituido con una estructura circular que mejora la solubilidad de itraconazol, un compuesto altamente lipofílico. Ello permite su aplicación endovenosa o aumentar la absorción de itraconazol y mejorar su biodisponibilidad oral ${ }^{28}$. La nueva presentación oral viene como SO y a diferencia de los preparados en cápsulas, debe ser ingerida sin alimentos para optimizar su absorción. La SO de itraconazol mejora el área bajo la curva (ABC), la concentración máxima (Cmáx) y el tiempo para alcanzar la concentración máxima (Tmáx) en comparación a la preparación en cápsulas ${ }^{28}$.

Se ha postulado que concentraciones plasmáticas de itraconazol $\geq 250-500 \mu \mathrm{g} / \mathrm{mL}$ son necesarias para asegurar una eficacia profiláctica y terapéutica, respectivamente ${ }^{31}$. Itraconazol SO permite mejorar la limitada absorción observada con las cápsulas y obtener niveles plasmáticos adecuados en pacientes con SIDA y hacerla independiente de la acidez gástrica. Con la SO se obtiene en esta población, concentraciones mínimas adecuadas después de cuatro días. Esta misma formulación ha permitido programar esquemas profilácticos en pacientes candidatos a trasplante de precursores hematopoyéticos (TPH) y obtener concentraciones presumiblemente terapéuticas durante los primeros ocho días de su aplicación y generalmente útiles en el momento del $\mathrm{TPH}$. Las principales diferencias entre la presenta- ción en cápsulas y la SO se presentan en la Tabla 7.

La formulación parenteral permite acelerar la obtención de concentraciones plasmáticas adecuadas estables, desde 14 días a sólo 2 días, cuando se utiliza un esquema de $200 \mathrm{mg}$ cada 12 horas por vía endovenosa durante 48 horas $^{28}$.

El compuesto itraconazol presenta una farmacocinética no lineal pero dosis dependiente. La concentración plasmática aumenta con el incremento de dosis pero más allá de lo esperado, presumiblemente por una saturación en el proceso de eliminación ${ }^{29,31}$. La SO no requiere ajustes en falla renal y la limitada experiencia en pacientes con insuficiencia hepática señala que los parámetros farmacocinéticos no se modifican en esta condición ${ }^{31}$. Itraconazol posee un metabolito activo (hidroxi-itraconazol) de similar actividad que el compuesto madre y que logra concentraciones algo superiores en plasma.

A pesar de que este compuesto logra muy bajas concentraciones en el LCR por su alto grado de unión a proteínas, se acumula lentamente en diferentes tejidos, habiendo sido utilizado con éxito en el tratamiento de algunas infecciones fúngicas del SNC. (ver más adelante) ${ }^{29}$

Efectos adversos e interacciones medicamentosas de itraconazol endovenoso o SO. La SO es bien tolerada. Las moléculas de ciclodextrina no son absorbidas y son metabolizados por bacterias intestinales en la glucosa, la que posteriormente se absorbe. Sin embargo, la sobrecarga de carbohidratos puede favorecer molestias gastrointestinales como náuseas, diarrea leve, vómitos o dolor abdominal. La frecuencia de interrupciones de tratamientos por efectos gastrointestinales severos oscila entre 3 y $8 \%$ siendo superior a lo observado con fluconazol oral ${ }^{11}$. La diarrea se observa con la presentación endovenosa u oral pero es más frecuente con la $\mathrm{SO}$ y proporcional a la dosis utilizada. Dosis mayores a $600 \mathrm{mg} /$ día de la SO se han asociado a insuficiencia suprarrenal, hipertensión y ginecomastia ${ }^{11}$. Las molestias relacionadas al preparado endovenoso son infrecuentes

Tabla 7. Principales diferencias entre la formulación de itraconazol en tabletas y la solución oral

\begin{tabular}{ll}
\hline Cápsulas & Solución oral \\
\hline - Biodisponibilidad limitada & $\bullet$ Mayor biodisponibilidad \\
- Absorción dependiente de pH ácido & - Independiente del pH ácido en estómago \\
- Requiere ser administrado después de las comidas & - Mejor absorción en ayunas \\
- Lenta obtención de concentraciones terapéuticas & - Acelera obtención de concentraciones terapéuticas \\
$(2$ semanas $)$ & $(4-8$ días $)$ \\
\hline
\end{tabular}


y generalmente del tipo flebitis en el sitio de punción. La molécula de hidroxipropil- $\beta$-ciclodextrina al ser aplicada por vía endovenosa se elimina por filtración glomerular y se acumula en casos de insuficiencia renal. Está contraindicada su administración endovenosa con clearance de creatinina menor a $30 \mathrm{ml} / \mathrm{min}$. En pacientes infectados por VIH se ha reportado una frecuencia elevada de casos de trombocitopenia.

Itraconazol presenta interacciones significativas con varios medicamentos comunes. Esta interacción es independiente de la formulación del fármaco y depende de la interacción específica con isoenzimas del complejo citocromo P-450 en el hígado (vide supra). Las concentraciones plasmáticas de itraconazol disminuyen con el uso concurrente de fenitoína, ranitidina o rifampicina reduciendo su efecto terapéutico ${ }^{33}$ (Tabla 3). Itraconazol SO e itraconazol en presentación parenteral no están disponibles aún en Chile.

Eficacia terapéutica. Diversos ensayos han evaluado la eficacia de las nuevas formulaciones de itraconazol en diferentes condiciones clínicas. Estas incluyen aspergilosis, infecciones por hongos endémicos, pacientes con episodios de neutropenia y fiebre y con esofagitis.

En aspergilosis se ha evaluado la eficacia de itraconazol endovenoso en forma abierta en un escaso número de pacientes, sin disponer aún de estudios comparativos con anfotericina B. Ha sido aplicado en dosis de $200 \mathrm{mg}$ endovenoso cada 12 horas durante 2 días, luego $200 \mathrm{mg}$ al día por 12 días adicionales y finalmente con terapia oral prolongada en dosis de $400 \mathrm{mg} / \mathrm{d}$. La tasa de respuesta completa o parcial alcanzó a $48 \%$ y en la mayor parte de los pacientes se lograron concentraciones plasmáticas mayores a $0,25 \mu \mathrm{g} / \mathrm{ml} \mathrm{a}$ pesar de que la fase oral fue desarrollada con cápsulas y no con la $\mathrm{SO}^{35}$. Los resultados terapéuticos con itraconazol endovenoso han sido inferiores que anfotericina B para la esterilización del LCR en el manejo de meningitis criptococóccica $^{35}$.

En pacientes con episodios de neutropenia y fiebre se evaluó itraconazol endovenoso versus anfotericina B en forma randomizada, con resultados similares para ambos grupos, de acuerdo a un conjunto de parámetros ( $53 \%$ para itraconazol y $46 \%$ para anfotericina B, diferencias no significativas). Éstos incluían la sobrevida a los 7 días de iniciado el tratamiento, desaparición de la fiebre durante la neutropenia, ausencia de una infección diseminada durante el tratamiento o hasta una semana de su término y el no retiro prematuro por intolerancia o toxicidad del medicamento. ${ }^{36}$ Itraconazol fue utilizado inicialmente como una dosis endovenosa de $200 \mathrm{mg}$ c/12 horas durante 48 horas, luego $200 \mathrm{mg}$ diarios con traspaso a una dosis de $400 \mathrm{mg}$ de SO desde el día 15 de tratamiento. La dosis de anfotericina B fue de 0,7 a $1 \mathrm{mg} / \mathrm{kg} / \mathrm{dí}^{36}$. El grupo con itraconazol se asoció a una mayor frecuencia de cambios de terapia antifúngica por persistencia de la fiebre, aunque estuvo asociado a una significativa menor tasa de efectos adversos, suspensión del tratamiento por toxicidad o reacciones adversas graves. La frecuencia de nefrotoxicidad con itraconazol fue de 0,5 versus $21 \%$ con anfotericina B. El grupo con itraconazol no se asoció a un mayor aumento de transaminasas en comparación al otro grupo, aunque sí a hiperbilirrubinemia $(10 \text { versus } 5 \%)^{36}$.

Utilidad clínica. En infecciones sistémicas, itraconazol endovenoso representa una alternativa a anfotericina $\mathrm{B}$ en el manejo de pacientes con aspergilosis invasora -especialmente en presencia de falla renal (aunque la formulación endovenosa está contraindicada con clearance de creatinina $<30 \mathrm{ml} / \mathrm{min}$ ) - en pacientes con infecciones por Candida no albicans que presentan un fracaso terapéutico ante fluconazol, debido a la mayor actividad de este compuesto para este tipo de aislados, y en pacientes oncológicos con episodios de neutropenia y fiebre bajo sospecha de una infección fúngica ${ }^{35}$.

La presentación en cápsulas no se considera una alternativa para el tratamiento de infecciones sistémicas o invasoras graves debido a su limitada biodisponibilidad, la lentitud para obtener concentraciones terapéuticas y la tolerancia limitada

Tabla 8. Resumen de las principales características de itraconazol

Disponibilidad de presentaciones con farmacocinética mejorada

Farmacocinética dosis-dependiente

Espectro de acción sobre levaduras y hongos filamentosos prevalentes en infecciones humanas

La SO no requiere ajustes en presencia de falla renal. Presentación ev contraindicada en falla renal moderada $(<30 \mathrm{ml} / \mathrm{min})$

Compuesto bien tolerado y no asociado a nefrotoxicidad

Interacciones con fármacos comunes al grupo de los triazólicos

Eficacia demostrada en pacientes con episodios de neutropenia y fiebre y sospecha de infección fúngica

Alto costo de adquisición para la presentación parenteral e intermedio para la SO

SO: solución oral 
que tiene el uso de altas dosis. Sin embargo, ha sido utilizada como un recurso desesperado y con éxito en numerosos casos.

En otras latitudes, el costo de adquisición diario de itraconazol endovenoso es más reducido que las formas lipídicas de anfotericina B pero superior al costo de fluconazol o anfotericina $\mathrm{B}$ tradicional..$^{10}$ En la Tabla 8 se presenta un resumen de las principales características de itraconazol.

\section{Voriconazol}

Este compuesto triazólico tiene un espectro de actividad más amplio que fluconazol e itraconazol y sólo es superado por anfotericina B (Tabla 5). $\mathrm{Su}$ espectro de actividad incluye aislados de Candida con resistencia adquirida (C. albicans, C. glabrata) o natural a fluconazol (C. krusei) y otras levaduras como C. neoformans, Trichosporon beigelii y Saccharomyces cerevisiae y es más activo que fluconazol e itraconazol sobre las principales levaduras de importancia clínica (Tabla 4$)^{1,7}$. Este compuesto es también activo contra diferentes especies de hongos filamentosos sobre los que ejerce un efecto fungicida dosis dependiente y que incluye al género Aspergillus (incluso A. terreus). También es activo contra hongos filamentosos endémicos (por ejemplo Histoplasma capsulatum), Pseudallescheria boydii y su forma sexual Scedosporium apiospermum, especies de Fusarium, Paecilomyces, Alternaria y Bipolaris (Tabla 5). Este compuesto no es activo contra los agentes de mucormicosis.

Aspectos farmacológicos. Al igual que itraconazol y fluconazol, este compuesto esta disponible en una presentación oral y otra endovenosa, tiene interacciones con fármacos similares a los otros compuestos y requiere una dosis de carga inicial para obtener concentraciones plasmáticas estables en el menor tiempo posible.

La presentación oral se encuentra disponible en tabletas de 50 y $200 \mathrm{mg}$ y su biodisponibilidad es mayor a $90 \%$ si se administra con las comidas, aunque su absorción no depende de un ambiente ácido en el estómago. La biodisponibilidad disminuye a $80 \%$ si se administra con alimentos grasos. Las dosis recomendadas para adultos corresponden a $400 \mathrm{mg}$ dos veces el primer día (en pacientes sobre $40 \mathrm{~kg}$ ) y luego $200 \mathrm{mg}$ cada 12 horas. (Tabla 2) Este esquema permite concentraciones plasmáticas de 2 a $3 \mu \mathrm{g} / \mathrm{ml}$.

La presentación endovenosa se administra en una dosis inicial de $6 \mathrm{mg} / \mathrm{kg}$ dosis cada 12 horas el primer día y a continuación una dosis de $4 \mathrm{mg} / \mathrm{kg}$ dosis cada 12 horas. Las concentraciones plasmáticas obtenidas con este esquema alcanzan a 3 a 6 $\mu \mathrm{g} / \mathrm{ml}$. La presentación parenteral está solubilizada en sulfobutil eter sódico de $\beta$-ciclodextrina (SBECD) y debe infundirse en 1 a 2 horas.

Las concentraciones en el LCR son aproximadamente $50 \%$ de las concentraciones plasmáticas pero las concentraciones tisulares en el SNC son superiores a las plasmáticas. Este fármaco es metabolizado en su mayor parte en el hígado y sólo 5\% se excreta en la orina sin modificaciones. Participan en su metabolismo los sistemas microsomales de la familia tipo 2 (CYP2C9 y CYP2C19) y tipo 3 (CYP3A4), siendo su vía principal la asociada a la isoenzima CYP2C19.

En pacientes con insuficiencia hepática leve a moderada se debe reducir la dosis de mantención a la mitad, pero no la dosis de carga. En pacientes con insuficiencia renal con clearance de creatinina menor a $50 \mathrm{ml} / \mathrm{min}$ está contraindicado el uso de voriconazol endovenoso (pero no la formulación oral) debido a la potencial acumulación tóxica de SBECD.

Las interacciones con otros medicamentos son numerosas y significativas debido a la metabolización común a través del sistema CYP-450. Las precauciones señaladas previamente para los triazólicos son aplicables a este compuesto, estando algunos fármacos contraindicados, ya sea porque son inductores del sistema microsomal y reducen las concentraciones plasmáticas de voriconazol o, porque este triazólico interfiere con el metabolismo de otros medicamentos prolongando su efecto o favoreciendo reacciones adversas (Tabla 3 ).

Efectos adversos. Este triazólico es en general bien tolerado, constituyendo las fotopsias el efecto adverso más característico y que ocurre en aproximadamente $30 \%$ de los pacientes. No deja lesiones permanentes y revierte en forma espontánea o al suspender el tratamiento. Las reacciones alérgicas cutáneas son la segunda manifestación adversa más frecuente, habitualmente leves y bien toleradas ( $~ 8 \%)$. Un aumento de las enzimas hepáticas se observa en aproximadamente 10 a $15 \%$ de los pacientes y puede superar los valores normales 3 a 5 veces $^{37}$. Este aumento, al parecer está asociado a altas concentraciones plasmáticas (> $6.000 \mu \mathrm{g} / \mathrm{ml}$ ), se resuelve con la suspensión del tratamiento y parece ocurrir con mayor frecuencia que lo observado con fluconazol.

Indicaciones para el uso de voriconazol en infecciones sistémicas. Voriconazol ha sido validado para el tratamiento de aspergilosis invasora siendo sus resultados mejores que los obtenidos con anfotericina $\mathrm{B}$ deoxicolato en términos de eficacia clínica y sobrevida a los 3 meses. ${ }^{38}$ En el estudio original, la mayor parte de los pacientes 
portaba neoplasias hematológicas y aspergilosis pulmonar, con una tasa de respuesta parcial o completa de $53 \%$ a las 12 semanas versus $32 \%$ observado con anfotericina B. Asimismo, el grupo con anfotericina B presentó una mayor frecuencia de efectos adversos graves (24 versus $13 \%)$.

Los resultados obtenidos en el tratamiento empírico de pacientes oncológicos con episodios de neutropenia y fiebre en comparación con anfotericina B liposomal, sugieren que el compuesto triazólico podría ser inferior a anfotericina $\mathrm{B}^{39}$. En sólo uno de los cinco componentes del sistema de comparación, voriconazol demostró ser superior a anfotericina B ("breakthrough infection" [98 versus $95,1 \%$ para anfotericina B]). El compuesto poliénico demostró una tendencia a un resultado superior en: sobrevida a los 7 días de finalizado el tratamiento [92 para voriconazol versus $94,1 \%$ para anfotericina B]; no discontinuación del tratamiento por toxicidad o por falta de eficacia antes de recuperar la neutropenia $(90,1$ versus $93,4 \%)$; resolución de la fiebre durante la neutropenia (32,5 versus $36,5 \%$ ); resolución completa o parcial de las infecciones establecidas al inicio. (46,2 versus $66,7 \%$ ) La respuesta global para voriconazol fue de 26 versus 30,6\% para anfotericina B liposomal con un intervalo de confianza $\mathrm{IC}_{95}$ de $-10,6$ a $1,5 \%$. El valor inferior de este intervalo $(-10,6 \%)$ supera en $0,6 \%$ el valor predefinido como indicador de un resultado no aceptable de inferioridad (10\% de diferencia). Por este motivo, la FDA estadounidense no aprobó este compuesto para esta condición. ${ }^{39-42}$

No existe hasta ahora una experiencia sólida en el uso de voriconazol para el tratamiento de pacientes con criptococosis meníngea. Voriconazol también ha sido validado para el tratamiento de hongos filamentosos como Fusarium spp. y para otros resistentes a anfotericina B como $P$. boydii. En la Tabla 9 se resumen las principales características asociadas a voriconazol.

\section{Análisis comparativo de los compuestos triazólicos}

Los estudios comparativos en el tratamiento de las infecciones fúngicas sistémicas han utilizado tradicionalmente como modelos a pacientes con candidiasis diseminada, meningitis criptococóccica, aspergilosis invasora y pacientes oncológicos con episodios de neutropenia y fiebre, y sospecha de infección fúngica. Este último caso es un factor de riesgo conocido para hongos filamentosos y el tratamiento empírico debe cubrir esta posibilidad (Tabla 10).
Tabla 9. Resumen de las principales características del voriconazol

Espectro de acción sobre levaduras y hongos filamentosos prevalentes en infecciones humanas

Elevada biodisponibilidad

Penetración al SNC ( $50 \%$ de concentraciones plasmáticas en el LCR y > al plasma en tejido cerebral)

Nuevas formulaciones que permiten acelerar obtención de concentraciones estacionarias

Absorción no dependiente de un pH gástrico ácido aunque disminuye con alimentos grasos

Farmacocinética no lineal en adultos

Contraindicación del preparado ev. cuando el clearance de creatinina es menor a $50 \mathrm{ml} / \mathrm{min}$ pero no del preparado oral

Eficacia clínica comprobada en aspergilosis invasora

Alto costo de adquisición

Itraconazol y voriconazol tienen actividad contra hongos filamentosos a diferencia de fluconazol, pero su espectro no incluye a los agentes de mucormicosis. Ambos preparados (itraconazol y voriconazol) son activos contra aislados de Candida no albicans con resistencia natural o adquirida a fluconazol.

Aunque estos tres preparados pueden ser utilizados teóricamente en infecciones por C. albicans, sólo fluconazol ha sido validado en este escenario, siendo además el único de ellos aprobado para el tratamiento de pacientes con meningitis criptococóccica de bajo riesgo. En pacientes con aspergilosis invasora sólo voriconazol ha sido formalmente comparado contra anfotericina B y se dispone de una evidencia menos sustantiva (no comparativa) sobre itraconazol endovenoso en esta condición. En contraste, en pacientes oncológicos con episodios de neutropenia y fiebre, con sospecha de infección fúngica, sólo itraconazol endovenoso ha demostrado un rendimiento semejante a anfotericina $\mathrm{B}$, lo que no pudo ser demostrado para voriconazol.

Los compuestos triazólicos se diferencian también en su frecuencia de administración, dosis, efecto del $\mathrm{pH}$ gástrico o de los alimentos sobre su absorción, tiempo para lograr concentraciones estacionarias y costo de adquisición. Los preparados parenterales de itraconazol y voriconazol no pueden ser administrados en pacientes con falla renal aunque el valor que contraindica su uso no es el mismo para ambos compuestos $(<30$ $v s<50 \mathrm{ml} / \mathrm{min}$ ) (Tabla 10). Los aspectos farma- 
Tabla 10. Cuadro comparativo entre diferentes compuestos triazólicos para el tratamiento de infecciones fúngicas diseminadas o invasoras

\begin{tabular}{|c|c|c|c|}
\hline Parámetro & Fluconazol & Itraconazol & Voriconazol \\
\hline \multicolumn{4}{|l|}{ Espectro de acción } \\
\hline Candida albicans & Sí & Sí & Sí \\
\hline Candida no albicans & Variable & Sí & Sí \\
\hline Cryptococcus neoformans & Sí & Sí & Sí \\
\hline Aspergillus spp. & No & Sí & Sí \\
\hline Otros hongos filamentosos & No & Variable & Variable \\
\hline Zygomycetes & No & No & No \\
\hline \multicolumn{4}{|l|}{ Aspectos farmacológicos } \\
\hline $\begin{array}{l}\text { Tiempo para lograr } \\
\text { concentraciones } \\
\text { estacionarias* }\end{array}$ & 24 horas & $\begin{array}{l}2 \text { semanas (cápsulas o SO) } \\
2 \text { días (vía ev) }\end{array}$ & 24 horas \\
\hline Biodisponibilidad & $\begin{array}{l}>90 \% \text { no afectada } \\
\text { por } \mathrm{pH} \text { gástrico }\end{array}$ & $\begin{array}{l}55 \%(\mathrm{SO}) \\
\text { SO debe ser } \\
\text { administrada en ayunas, } \\
\text { el incremento del pH } \\
\text { limita absorción de la } \\
\text { presentación en cápsulas }\end{array}$ & $\begin{array}{l}>90 \% \text { no afectada } \\
\text { por } \mathrm{pH} \text { gástrico }\end{array}$ \\
\hline $\begin{array}{l}\text { Concentraciones en } \\
\text { LCR o SNC }\end{array}$ & LCR $>70 \%$ plasma & LCR $1 \%$ & $\begin{array}{l}\text { LCR } 50 \% \text { plasma } \\
\text { SNC > plasma (en tejidos) }\end{array}$ \\
\hline Ajuste de dosis en falla renal & Sí & $\begin{array}{l}\text { Contraindicado uso ev con } \\
\mathrm{Cl} \text { creatinina }<30 \mathrm{ml} / \mathrm{min} \text {, } \\
\text { las presentaciones orales } \\
\text { no requieren ajuste de dosis }\end{array}$ & $\begin{array}{l}\text { Contraindicado uso ev con } \\
\mathrm{Cl} \text { creatinina }<50 \mathrm{ml} / \mathrm{min} \text {, } \\
\text { presentación oral no } \\
\text { requiere ajuste de dosis }\end{array}$ \\
\hline $\begin{array}{l}\text { Ajuste de dosis en falla } \\
\text { hepática }\end{array}$ & No aplicable & Datos no disponibles & $\begin{array}{l}1 / 2 \text { dosis de mantención por } \\
\text { vía oral }(100 \mathrm{mg} \mathrm{c} / 12 \mathrm{~h})\end{array}$ \\
\hline \multicolumn{4}{|l|}{ Eficacia clínica } \\
\hline Candidiasis diseminada $* *$ & Sí & No documentado & No documentado \\
\hline Meningitis criptococóccica & $\begin{array}{l}\text { Como terapia de inducción } \\
\text { en pacientes de bajo riesgo }\end{array}$ & $\begin{array}{l}\text { Inferior a anfotericina B } \\
\text { en terapia de inducción o } \\
\text { en profilaxis secundaria }\end{array}$ & No documentado \\
\hline $\begin{array}{l}\text { Episodios de neutropenia } \\
\text { y fiebre en pacientes con } \\
\text { cáncer }\end{array}$ & $\begin{array}{l}\text { No recomendado por } \\
\text { espectro limitado }\end{array}$ & $\begin{array}{l}\text { Respuesta similar a } \\
\text { anfotericina para el } \\
\text { preparado ev }\end{array}$ & $\begin{array}{l}\text { Respuesta inferior a } \\
\text { anfotericina B liposomal }\end{array}$ \\
\hline Aspergilosis invasora & $\begin{array}{l}\text { No recomendado por } \\
\text { espectro limitado }\end{array}$ & $\begin{array}{l}\text { Respuesta favorable } \\
\text { en } 48 \%\end{array}$ & $\begin{array}{l}\text { Respuesta superior a } \\
\text { anfotericina B }\end{array}$ \\
\hline \multicolumn{4}{|l|}{ Otros aspectos } \\
\hline Costo de adquisición & Bajo & Intermedio & Alto \\
\hline
\end{tabular}

*: utilizando dosis de carga inicial; **: en pacientes no neutropénicos 
coeconómicos asociados al uso de triazoles se incluyen en la segunda parte de esta serie.

\section{Bibliografía}

1.- Ghannoum M A, Kuhn D M. Voriconazole. Better chances for patients with invasive mycosis. Eur J Med Res 2002; 7: 242-56.

2.- Pea F, Furlanut M. Pharmacokinetic aspects of treating infections in the intensive care unit. Focus on drug interactions. Clin Pharmacokinet 2001; 40: 833-68.

3.- Albengres E, Le Louet H, Tillement JP. Systemic antifungal agents. Drug interactions of clinical significance. Drug Saf 1998; 18: 83-97.

4.- Finch C, Green C A, Self T H. Fluconazolecarbamazepine interaction. South Med J 2002; 95: 1099-100.

5.- Nair D R, Morris H H. Potential fluconazole-induced carbamazepine toxicity. Ann Pharmacother 1999; 33: 790-2.

6.- Jaruratanasirikul S, Sriwiriyajan S. Effect of omeprazole on the pharmacokinetics of itraconazole. Eur J Clin Pharmacol 1998; 54: 159-61.

7.- Johnson L B, Kaufmann C A. Voriconazol: A new triazole agent. Clin Infect Dis 2003; 36: 630-7.

8.- Stone E A, Fung H B, Kirschenbaum H L. Caspofungin: an echinocandin antifungal agent. Clin Ther 2002; 24: 351-77.

9.- Espinel-Ingroff A. Comparison of in vitro activities of the new triazole SCH 56592, and the echinocandins MK-0991 (L 743,872) and LY303366 against opportunistic filamentous and dismorphic fungi and yeasts. J Clin Microbiol 1998; 36: 2950-56.

10.- Pacetti S A, Gelone S P. Caspofungin acetate for treatment of invasive fungal infections. Ann Pharmacother 2003; 37: 90-8.

11.- Martin M V. The use of fluconazole and itraconazole in the treatment of Candida albicans infections: A review. J Antimicrob Chemother 1999; 44: 429-37.

12.- Silva V, Diaz M C, Febré N. Red de Diagnóstico en Micología Médica. Vigilancia de la resistencia de levaduras a antifúngicos. Rev Chil Infect 2002; 19 (Supl 2): S149-56.

13.- Calvo B M, Colombo A L, Fischman O et al. Antifungal susceptibilities varieties, and electrophoretic karyotypes of clinical isolates of Cryptococcus neoformans from Brazil, Chile, and Venezuela. J Clin Microbiol 2001; 39: 2348-50.

14.- Muhl E, Martens T, Iven H, Rob P, Bruch H P. Influence of continuous veno-venous haemodiafiltration and continuous veno-venous haemofiltration on the pharmacokinetics of fluconazole. Eur J Clin Pharmacol 2000; 56: 671-8.

15.- Pelz R K, Lipsett P A, Swoboda S M, Merz W, Rinaldi M G, Hendrix V W. Enteral fluconazole is well absorbed in critically ill surgical patients. Surgery 2002; 131: 534-40.

16.- Davis G A, Rapp R P, Kearney P A, Ensom M H H. Pharmacokinetics of fluconazole in trauma patients with postpyloric feeding tubes. Ann Pharmacother 2001; 35: 1492-4.

17.- Buijk SLCE, Gyssens I C, Mouton J W, Verbrugh H A, Touw D J, Bruining H A. Pharmacokinetics of sequential intravenous and enteral fluconazole in critically ill surgical patients with invasive mycoses and compromised gastro-intestinal function. Intensive
Care Med 2001; 27: 115-21.

18.- Rajagopalan P, Pelz R K, Lipsett P A, Swoboda S M, Rinaldi M G, Hendrix C W. Enteral fluconazole population pharmacokinetics in patients in the surgical intensive care unit. Pharmacother 2003; 23: 592-602.

19.- Rex J H, Bennett J E, Sugar A M et al. A randomized trial comparing fluconazole with amphotericin B for the treatment of candidemia in patients without neutropenia. N Engl J Med 1994; 331: 1325-30

20.- Rex J H, Pappas P G, Karchmer A W et al. A randomized and blinded multicenter trial of high-dose fluconazole plus placebo versus fluconazole plus amphotericin B as therapy for candidemia and its consequences in non neutropenic subjects. Clin Infect Dis 2003 ; 36: 1221-8

21.- Van der Horst C M, Saag M S, Cloud G A et al. Treatment of cryptococcal meningitis associated with the acquired immunodeficiency syndrome. N Engl J Med 1997; 337: 15-21

22.- Saag M S, Powderly W G, Cloud G A et al. Comparison of amphotericin B with fluconazole in the treatment of acute AIDS-associated cryptococcal meningitis. N Engl J Med 1992; 326: 83-9

23.- Haubrich R H, Haghighat D, Bozzette S A, Tilles J, McCutchan J A, California Collaborative Treatment Group. High-dose fluconazole for treatment of cryptococcal disease in patients with human immunodeficiency virus infection. J Infect Dis 1994; 170: 238-42.

24.- Powderly W G, Saag M S, Cloud G A et al. A controlled trial of fluconazole or amphotericin B to prevent relapse of cryptococcal meningitis in patients with the acquired immunodeficiency syndrome. N Engl J Med 1992; 326: 793-8.

25.- Rex J H, Walsh T J, Sobel J D et al. Practice guidelines for the treatment of candidiasis. Clin Infect Dis 2000; 30: 662-78.

26.- Czwerwiec F S, Bilsker M S, Kamerman M L, Bisno A L. Long-term survival after fluconazole therapy of candidal prosthetic valve endocarditis. Am J Med 1993; 94: $545-6$

27.- Matsumoto K, Ueno K, Yoshimura $\mathrm{H}$ et al. Fluconazole-induced convulsions at serum through concentrations of approximately $80 \mu \mathrm{g} / \mathrm{ml}$. Ther Drug Monitor 2000; 22: 635-6

28.- Willems L, van der Geest R, de Beule K. Itraconazole oral solution and intravenous formulations: a review of pharmacokinetics and pharmacodynamics. J Clin Pharm Ther 2001; 26: 159-69.

29.- Grant S M, Clissold S P. Itraconazole. A review of its pharmacodynamic and pharmacokinetic properties, and therapeutic use in superficial and systemic mycoses. Drugs 1989; 37: 310-44.

30.- Schafer-Korting M. Pharmacokinetic optimisation of oral antifungal therapy. Clin Pharmacokinet 1993; 25: 329-41

31.- Poirier J M, Cheymol G. Optimisation of itraconazole therapy using target drug concentrations. Clin Pharmacokinet 1998; 35: 461-73.

32.- Negroni R, Arechavala AI. Itraconazole: Pharmacokinetics and indications. Arch Med Res 1993; 24: 387-93.

33.- Denning D W, Lee J Y, Hostetler J S et al. NIAID Mycoses Study Group multicenter trial of oral itraconazole therapy for invasive aspergillosis. Am J Med 1994; 97: 135-44.

34.- Stevens D A, Lee J Y. Analysis of compassionate use 
of itraconazole therapy for invasive aspergillosis by the NIAID Mycoses Study Group criteria. Arch Intern Med 1997; 157: 1857-62.

35.- Slain D, Rogers P D, Cleary J D, Chapman S W. Intravenous itraconazole. Ann Pharmacother 2001; 35: 720-9.

36.- Boogaerts M, Winston D J, Bow E J et al. Intravenous and oral itraconazole versus intravenous amphotericin $\mathrm{B}$ deoxycholate as empirical antifungal therapy for persistent fever in neutropenic patients with cancer who are receiving broad-spectrum antibacterial therapy. Ann Intern Med 2001; 135: 412-22.

37.- Potoski B A, Brown J. The safety of voriconazole. Clin Infect Dis 2002; 35: 1273-5

38.- Herbrecht R, Denning D W, Patterson T F et al. Voriconazole versus amphotericin B for primary therapy of invasive aspergillosis. N Engl J Med 2002; 347: 408-15.

39.- Walsh T J, Pappas P, Winston D J et al. Voriconazole compared with liposomal amphotericin B for empirical antifungal therapy in patients with neutropenia and persistent fever. N Engl J Med 2002; 346: 225-34

40.- Walsh T J, Lee J, Dismukes W E. Decision about voriconazole versus liposomal amphotericin B [letter]. N Engl J Med 2002; 346: 1499.

41.- Powers J H, Dixon C A, Goldberger M J. Voriconazole versus liposomal amphotericin B in patients with neutropenia and persistent fever [letter]. N Engl J Med 2002; 346: 289-90

42.- Ullman A J, Heussel C P, Cornely O A. Voriconazole versus liposomal amphotericin $\mathrm{B}$ for empirical antifungal therapy [letter]. N Engl J Med 2002; 346: 1745-7.

Correspondencia:

Alberto Fica Cubillos

E-mail: afica@ns.hospital.uchile.cl 